\section{Estudo Multicêntrico de Pacientes Brasileiros Com Deficiência da 21-Hidroxilase: Correlação do Genótipo Com o Fenótipo}

\begin{abstract}
RESUMO
Analisamos as características clínicas e moleculares de 205 pacientes portadores das diferentes formas clínicas da deficiência da 21 -hidroxilase, com diagnóstico hormonal e molecular definidos. As mutações mais freqüentes foram a 12 splice na forma perdedora de sal, a I172N na forma virilizante simples e a V281L na forma não clássica, com freqüências semelhantes às de outros estudos. Obtivemos baixa freqüência de deleção do gene da 21-hidroxilase, de forma semelhante ao identificado nas populações argentina e mexicana. Cinco mutações novas foram descritas em nossa população: G424S, H28+C, Ins $1003^{\wedge} 1004$ A, R408C e IVS2-2A>G. A severidade do genótipo também se correlacionou diretamente com níveis mais elevados de $170 \mathrm{OH}$-progesterona e de testosterona. As mutaçōes foram classificadas em três grupos, de acordo com o comprometimento da atividade enzimática observado in vitro: Grupo A: atividade de 0-2\%; Grupo B: atividade de 3$7 \%$ e Grupo C: atividade $>20 \%$. Houve forte correlação do grupo A com a forma perdedora de sal, do grupo $B$ com a forma virilizante simples e do grupo $\mathrm{C}$ com a forma não clássica. A mutação 12 splice (Grupo A) em homo ou hemizigose conferiu o fenótipo de forma clássica, embora tanto a forma perdedora de sal quanto a forma virilizante simples tenham sido identificadas. A boa correlação do genótipo com o fenótipo na HAC-210H permite sua aplicação na prática clínica, para o aconselhamento genético, diagnóstico e tratamento pré-natal das gestações de risco para a forma clássica da HAC-21OH e para confirmação diagnóstica após screening neonatal da HAC-210H, exceto na presença da mutação I2splice. (Arq Bras Endocrinol Metab 2004;48/5:697-704)
\end{abstract}

Descritores: Deficiência da 21-hidroxilase; Mutações; Genes CYP21A2; Genótipo e fenótipo; Estudo multicêntrico

\begin{abstract}
Multicentric Study of Brazilian Patients With 21-Hydroxylase Deficiency: A Genotype-Phenotype Correlation.

We analyzed the clinical and molecular data of 205 patients with the three different clinical forms of 21-hydroxylase deficiency, in whom the clinical and molecular diagnosis were already defined. The most frequent mutations were I2 splice in the salt wasting form, I172N in the simple virilizing and V281L in the nonclassical form, presenting similar frequencies as those observed in other populations. We found a lower frequency of 21-hydroxylase gene deletion, similar to that previously identified in Argentinean and Mexican populations. Five new mutations were described in our population: G424S, H28+C, Ins $1003^{\wedge} 1004$ A, R408C and IVS2-2A>G. The genotype was classified in three groups according to the impairment of enzymatic activity observed in vitro, Group A: 0-2\%, Group B: $3-7 \%$ and Group C: $>20 \%$. Group A mutations correlated with the salt wasting form, the Group B with simple virilizing form and Group $C$ with the non classical form. The severity of genotype showed a positive cor-
\end{abstract}

artigo original

Tânia A.S.S. Bachega

Ana Elisa C. Billerbeck

Érica B. Parente

Sofia H.V. Lemos-Marini

Maria Tereza M. Baptista

Maricilda P. Mello

Gil Guerra Jv.

Hilton Kuperman

Nuvarte Setian

Durval Damiani

Natália Torres

Margaret de Castro

Berenice B. de Mendonça

Laboratório de Hormônios e Genética Molecular (TASSB, AECB, EBP, $B B M)-L I M / 42$, Disciplina de Endocrinologia do Departamento de

Clínica Médica do Hospital das Clinicas, e Unidade de

Endocrinologia Pediátrica do

Instituto da Criança (HK, NS, DD) do Hospital das Clínicas da

Faculdade de Medicina da

Universidade de São Paulo,

São Paulo; Centro de Biologia Molecular e Engenharia Genética (SHVL-M, MTMB, MPM, GGJ),

Departamento de Pediatria,

Centro de Investigação em

Pediatria e Disciplina de

Endocrinologia da Faculdade de Ciências Médicas da Universidade

Estadual de Campinas,

Campinas; e Departamento de Clínica Médica (NT, MC) da Facul dade de Medicina de Ribeirão Preto, Universidade de São Paulo, Ribeirão Preto, SP.

Recebido em 27/08/04 Aceito em 02/09/04 
relation with higher $17 \mathrm{OH}$-progesterone and testosterone levels. The 12 splice mutation in homo or hemizygosis confers classical form phenotype with both salt wasting and simple virilizing forms, precluding the prediction of the clinical form through genotype in pre and neonatal diagnosis. The good genotypephenotype correlation in patients with 21 -hydroxylase deficiency shows the usefulness of genotype to predict the clinical form for genetic counseling, prenatal diagnosis and to confirm neonatal screening diagnosis, except in cases with 12 splice mutation. (Arq Bras Endocrinol Metab 2004;48/5:697-704)

Keywords: 21-Hydroxylase deficiency; Mutations; CYP21A2 Gene; Genotype and phenotype; Multicentric study

A HIPERPLASIA ADRENAL CONGÊNITA é um dos mais freqüentes erros herdados do metabolismo, sendo a deficiência da enzima 21-hidroxilase (HAC-21OH) responsável por $90 \%$ a $95 \%$ dos casos $(1,2)$.

A HAC-21OH apresenta diferentes manifestações clínicas que incluem uma forma severa (clássica) com virilização pré-natal da genitália externa em fetos femininos e pós-natal em ambos os sexos, com ou sem perda de sal, e uma forma mais leve em que os sintomas se iniciam mais tardiamente (não clássica) através de pubarca precoce, alterações menstruais, hirsutismo, infertilidade ou podendo também ser assintomática (1). Estas diferentes formas clínicas representam um contínuo espectro do comprometimento da atividade da 21hidroxilase, causado por mutações no gene que codifica a enzima.

O hormônio marcador do diagnóstico da HAC$210 H$ é a $17 \mathrm{OH}$-progesterona, que na forma clássica geralmente está acima de $60 \mathrm{ng} / \mathrm{mL}$. Na forma não clássica, o critério correntemente utilizado é o valor basal da $17 \mathrm{OH}$-progesterona $>5 \mathrm{ng} / \mathrm{mL}$ (3) ou pósestímulo com ACTH $>10 \mathrm{ng} / \mathrm{mL}$, definido antes dos estudos moleculares da HAC-210H (4).

\section{Genética Molecular da Deficiência da 21-Hidroxilase}

$O$ gene que codifica a enzima 21-hidroxilase, denominado CYP21A2, está localizado no braço curto do cromossomo 6, juntamente com o seu pseudogene altamente homólogo CYP2IAIP e alternam-se em tandem com os genes $C 4 A$ e $C 4 B$, que codificam o quarto componente do complemento sérico (5-7). A alta homologia entre os genes CYP21 e a organização dos genes duplicados em cadeia nesta região do cromossomo favorecem o emparelhamento desigual durante a meiose e, conseqüentemente, a ocorrência de eventos de cross - ing over desiguais, podendo gerar alelos duplicados ou deletados. Através do emparelhamento desigual destes genes, também podem ocorrer eventos de conversão gênica, com transferência de seqüências deletérias do pseudogene para o gene ativo (8).

As mutações mais freqüentes na HAC-21OH são as de ponto. Mais de 65 mutações de ponto foram descritas no gene CYP21A2, das quais 15 são mutações também encontradas no pseudogene, que são transferidas para o gene ativo através de processos de micro-conversão gênica, enquanto que as demais representam eventos mutagênicos casuais (9).

Os grandes rearranjos gênicos são geralmente identificados através de metodologias como o Southern blotting, seguido de hibridação com sonda específica para o gene CYP21A2. As mutações de ponto, já conhecidas, podem ser identificadas por técnicas tais como a reação de polimerização em cadeia (PCR) alelo-específico ou PCR seguido de hibridação com sondas de oligonucleotídeos específicas $(10,11)$.

\section{Freqüência das Mutaçōes na População Brasileira com HAC-21OH}

É importante a determinação da freqüência das mutações em cada população, pois estas podem variar de acordo com o grupo étnico. Analisamos a freqüência destas mutações numa amostra de 205 pacientes brasileiros. Estes pacientes são provenientes da Unidade de Endocrinologia do Hospital das Clínicas da FMUSP (n= 104), da Unidade de Endocrinologia Pediátrica do Instituto da Criança da FMUSP $(n=13)$, do Departamento de Clínica Médica do Hospital das Clínicas da Faculdade de Medicina de Ribeirão Preto $(\mathrm{n}=13)$ e do Ambulatório de Endocrinologia Pediátrica da Universidade Estadual de Campinas $(n=75)$. Estes pacientes foram selecionados após a confirmação do diagnóstico hormonal de HAC-21OH e do diagnóstico molecular, isto é, a identificação de mutações em ambos os alelos.

Considerando-se a forma clínica da doença, 89 pacientes são portadores da forma perdedora de sal, sendo 64 do sexo feminino e 25 do sexo masculino, 71 são portadores da forma virilizante simples, sendo 40 do sexo feminino e 31 do sexo masculino, e 45 portadores da forma não clássica, sendo 43 do sexo feminino e 2 do sexo masculino. Como o padrão da herança desta doença é autossômico recessivo, espera-se igual número de afetados entre os sexos. No entanto, o número menor de indivíduos do sexo masculino nesta amostra sugere que os homens não estão sendo diagnosticados no nosso meio, e que meninos com a forma perdedora de sal devam ir a óbito no período neonatal 
por crise de perda de sal, pois a incidência da forma perdedora de sal corresponde a $2 / 3$ dos casos da forma clássica em diferentes casuísticas (12).

Os grandes rearranjos gênicos estão presentes em 20 a 33\% dos alelos em pacientes de origem caucasiana (12). Observamos que os grandes rearranjos, deleção do CYP21A2 e grande conversão gênica, foram identificados em apenas $13 \%$ dos alelos. Nossos pacientes, de forma semelhante ao que ocorre em pacientes de origem mexicana e argentina $(13,14)$, apresentaram uma freqüência mais baixa de deleções do CYP21A2 (tabela 1). Os demais alelos apresentam mutações de ponto, e 90 a $95 \%$ destes alelos apresentam as mutações que normalmente são encontradas no pseudogene $(10,15-17)$.

As diferentes mutações do gene CYP21A2 foram correlacionadas com as formas de apresentação clínica. Mutações que alteram a rede de leitura, que alteram os sítios de splicing ou que criam códons de parada de leitura são encontradas em pacientes com a forma perdedora de sal, já as mutações do tipo mis sense, que substituem aminoácidos, dependendo da sua posição na proteína, são encontradas nas diferentes formas clínicas da HAC-210H.

Na nossa casuística, a mutação mais freqüente na forma perdedora de sal foi a I2 splice presente em $55 \%$ dos alelos, na forma virilizante simples a $1172 \mathrm{~N}$ presente em $42 \%$ dos alelos, seguida pela 12 splice em $25 \%$ dos alelos e a V281L na forma não clássica presente em $70 \%$ dos alelos (tabela 1). Obtivemos freqüência mais baixa das mutações P30L e P453S, associadas à forma não clássica, do que a descrita na literatura (12). Observamos uma associação significante da mutação I2 splice com a forma perdedora de sal, da mutação I172N com a forma virilizante simples e da mutação V281L com a forma não clássica. A despeito da diversidade étnica da população brasileira, a freqüência das mutações de ponto em nossa população foi semelhante à da maioria dos estudos populacionais (10,15-17) (tabela 2).

Observamos a ocorrência de duas ou mais mutações de ponto no mesmo alelo em $7 \%$ dos alelos. A identificação de duas mutações no DNA de um paciente não é suficiente para se concluir o diagnóstico molecular, e para adequada genotipagem é necessário realizar a segregação destas mutações no DNA dos pais do paciente. As combinações de mutações por alelo mais freqüentes foram Ins $\mathrm{T}+$ Q318X + R356W, Q318X + R356W, I2 splice + V281L. As duas primeiras combinações contêm mutações que são adjacentes no pseudogene e provavelmente foram transferidas como um único segmento para o gene ativo. As mutações I2 splice + V281L não são contíguas e provavelmente foram transferidas em dois processos independentes de micro-conversão. Assim, observamos que qualquer segmento do pseudogene pode ser transferido para o gene ativo.

O estudo dos grandes rearranjos e das mutações de ponto derivadas do pseudogene identificou mutações em 76 a 93\% dos alelos na população brasileira com as formas clássica e não clássica da deficiência da 21 hidroxilase (18-22), indicando que os demais alelos devem conter mutações novas.

O seqüenciamento do gene CYP2IA2 foi realizado em pacientes com genótipo incompleto e identificou mutações novas. A primeira foi a mutação G424S encontrada em 4 pacientes com a forma virilizante simples. Todos os indivíduos portadores desta mutação apresentavam, no mesmo alelo, a deleção dos genes $C 4 A$ e CYP21A1P, além da presença do HLA DR17, sugerindo um efeito de gene fundador (23). Posteriormente, foi identificada a mutação $\mathrm{H} 28+\mathrm{C}$ em homozigose em paciente proveniente de casamento consangüíneo, portadora da forma perdedora de sal (24). A inserção deste nucleotídeo no códon 28 altera a rede de leitura e cria um códon de parada de leitura no aminoácido 78 . Outras mutações foram descritas como a $1003^{\wedge} 1004$ Ins A, R408C e IVS2$2 \mathrm{~A}>\mathrm{G}$, identificadas em 11 pacientes com a forma clássica (25). Os indivíduos portadores das mesmas mutações possuíam os mesmos haplótipos em estudo de microssatélites, sugerindo também que estas três mutações são provenientes de um gene fundador. Quatro das cinco mutações novas identificadas na população brasileira apresentam efeito de gene fundador, e a sua pesquisa em outros grupos étnicos pode traçar a origem da nossa população.

Tabela 1. Freqüência (\%) das mutações derivadas do pseudogene em pacientes brasileiros com as diferentes formas clínicas da deficiência da 21-hidroxilase.

\begin{tabular}{|c|c|c|c|c|c|c|c|c|c|c|c|c|}
\hline $\begin{array}{c}\text { Alelos } \\
\text { (n) }\end{array}$ & Forma Clínica & G.R. & P30L & $12 \mathrm{sp}$ & Del 8 nt & $1172 N$ & Cluster & V281L & Ins T & Q318X & R356W & P453S \\
\hline $\begin{array}{l}178 \\
142 \\
90\end{array}$ & $\begin{array}{l}\text { PS } \\
\text { VS } \\
\text { NC }\end{array}$ & $\begin{array}{c}19 \\
10,5 \\
5,5\end{array}$ & $\begin{array}{l}- \\
0,7 \\
4,4\end{array}$ & $\begin{array}{l}55 \\
25 \\
6,6\end{array}$ & $\begin{array}{l}1,7 \\
0,7 \\
3,3\end{array}$ & $\begin{array}{c}- \\
42 \\
2,2\end{array}$ & $\begin{array}{l}- \\
-\end{array}$ & $\begin{array}{l}5,6 \\
0,7 \\
70\end{array}$ & $\begin{array}{l}5,6 \\
2,8 \\
-\end{array}$ & $\begin{array}{l}14 \\
11 \\
-\end{array}$ & $\begin{array}{c}9,5 \\
13,4 \\
6,6\end{array}$ & $\begin{array}{c}- \\
- \\
4,4\end{array}$ \\
\hline
\end{tabular}

PS: perdedor de sal, VS: virilizante simples, NC: não clássica, G.R.: grande rearranjo, sp: splice, Del: deleção 
Tabela 2. Freqüência (\%) das mutações de ponto de acordo com o número total de alelos em diferentes populações com a forma clássica e não clássica da deficiência da 21-hidroxilase.

\begin{tabular}{|c|c|c|c|c|c|c|c|c|c|c|c|c|}
\hline $\begin{array}{l}\text { País } \\
\text { (n) }\end{array}$ & Alelos & P30L & $12 \mathrm{sp}$ & Del 8 nt & $1172 N$ & Cluster & V281L & Ins T & Q318X & R356W & P453S & Referências \\
\hline $\begin{array}{l}\text { Suécia } \\
\text { França } \\
\text { EUA } \\
\text { Espanha } \\
\text { Argentina } \\
\text { Itália } \\
\text { México } \\
\text { Brasil }\end{array}$ & $\begin{array}{c}186 \\
182 \\
158 \\
76 \\
72 \\
146 \\
94 \\
410\end{array}$ & $\begin{array}{c}1,6 \\
\star \\
2,5 \\
2,6 \\
\star \\
8,2 \\
8,5 \\
1\end{array}$ & $\begin{array}{c}30,5 \\
20 \\
26 \\
25 \\
18 \\
29,4 \\
47 \\
34\end{array}$ & $\begin{array}{c}1,2 \\
7 \\
10 \\
4 \\
2,7 \\
1,4 \\
2,1 \\
1,7\end{array}$ & $\begin{array}{c}20,8 \\
4 \\
15,8 \\
1,3 \\
15,3 \\
9,6 \\
11,7 \\
14\end{array}$ & $\begin{array}{l}1,1 \\
11 \\
6,3 \\
0 \\
0 \\
0 \\
0 \\
0\end{array}$ & $\begin{array}{c}7 \\
15 \\
8,9 \\
18,4 \\
* \\
15,8 \\
8,5 \\
18\end{array}$ & $\begin{array}{c}0,5 \\
1 \\
2,5 \\
1,3 \\
* \\
0,7 \\
1,1 \\
3\end{array}$ & $\begin{array}{c}3,2 \\
\star \\
5,7 \\
4 \\
13,8 \\
10,3 \\
4,3 \\
7,3\end{array}$ & $\begin{array}{c}4,3 \\
\star \\
7,6 \\
4 \\
5,5 \\
0,7 \\
7,4 \\
11\end{array}$ & $\begin{array}{c}0,5 \\
\star \\
* \\
* \\
* \\
* \\
2,1 \\
1\end{array}$ & $\begin{array}{c}11 \\
15 \\
10 \\
16 \\
14 \\
17 \\
38 \\
\text { Presente } \\
\text { Estudo }\end{array}$ \\
\hline
\end{tabular}

*: mutação não pesquisada, sp: splice, Del: deleção

A técnica de seqüenciamento permitiu identificar mutações em 100\% dos alelos da forma clássica, enquanto que a forma não clássica permanece com $88 \%$ dos alelos sem mutações identificadas. Este último fato também é observado em outros estudos populacionais que incluíram em sua amostra número significativo de pacientes com a forma não clássica $(15,16,26)$.

\section{Correlação do Genótipo com o Fenótipo}

A alta freqüência de pacientes com a HAC-21OH permite correlacionar as diferentes manifestações clínicas da doença com a atividade enzimática conferida por cada mutação observada em estudos de mutagênese e expressão in vitro (tabela 3). Speiser e cols. (10) dividiram as mutações em grupos de acordo com o comprometimento da atividade enzimática. O grupo A incluiu as mutações que abolem a atividade enzimática, subgrupo Al: grandes rearranjos, deleção de 8 nucleotídeos no éxon 3, cluster, Ins T, R356W, Q318X e subgrupo A2: a mutação I2 splice que confere $<2 \%$ de atividade in vitro. $\mathrm{O}$ grupo B incluiu a mutação $1172 \mathrm{~N}$ que confere 3 a $7 \%$ de atividade enzimática. $\mathrm{O}$ grupo $\mathrm{C}$ incluiu as mutações V281L e P30L, que conferem mais que $20 \%$ de atividade enzimática. Os autores observaram que indivíduos homozigotos para mutações do grupo A apresentaram principalmente a forma perdedora de sal, homozigotos para a mutação do grupo B ou em heterozigose composta com as mutações do grupo A apresentaram a forma virilizante simples, e os homozigotos para mutações do grupo $\mathrm{C}$ ou em heterozigose composta com as anteriores apresentaram a forma não clássica. Foi observado que a forma clínica em um indivíduo heterozigoto composto, isto é, mutações diferentes entre os alelos, é definida pelo alelo que apresenta maior atividade enzimática.

Analisando a nossa amostra de pacientes com genótipo definido, observamos que 39\% eram homozigotos para uma determinada mutação e os demais heterozigotos compostos. Cento e dez pacientes apresentaram genótipo do grupo A (35 subgrupo Al e 75 subgrupo A2), 46 do grupo B e 49 do grupo C. Os genótipos mais freqüentes estão descritos na tabela 4 . Entre os pacientes com genótipo do subgrupo $\mathrm{Al} / \mathrm{Al}, 23$ apresentaram a forma perdedora de sal e 12 a forma virilizante simples e no subgrupo A2, 62 pacientes apresentaram a forma perdedora de sal e 13 a forma virilizante simples. A forma virilizante simples em pacientes com genótipo do grupo A esteve relacionada principalmente com a presença das mutações R356W e I2 splice.

Todos os pacientes com o genótipo do grupo B, exceto um (genótipo I172N/V281L), apresentaram a forma virilizante simples, de forma semelhante ao observado em outros estudos populacionais $(10,15$ 17). Em trabalho de origem finlandesa (27), este genótipo foi também associado à forma perdedora de sal, embora os mecanismos que justificassem esta associação não tenham sido esclarecidos.

Os pacientes com genótipo do grupo $\mathrm{C}$, exceto quatro, apresentaram a forma não clássica. Destes quatro pacientes, três apresentavam a forma perdedora de sal e um a forma virilizante simples, e os seus genótipos continham um alelo com mutação do grupo A e o outro alelo com a mutação V281L do grupo C. Entretanto, como o DNA destes pacientes não foi seqüenciado, não podemos afastar a existência de uma segunda mutação no alelo que contem a mutação V281L. Casos com genótipos e fenótipos semelhantes a estes 4 pacientes foram descritos previamente (28), e o posterior seqüenciamento do DNA identificou uma nova mutação em todos os casos no alelo contendo a mutação V281L (dados não publicados).

Analisamos, nesta amostra, outros dados clínicos e laboratoriais dos diferentes genótipos. A média dos valores basais de 17-hidroxiprogesterona e da testosterona nos diferentes grupos genotípicos correlacionou-se com a severidade do comprometimento da

Arq Bras Endocrinol Metab vol $48 n^{\circ} 5$ Outubro 2004 
Tabela 3. Mutações mais freqüentes encontradas na deficiência da 21-hidroxilase agrupadas de acordo com o comprometimento da atividade enzimática observado in vitro.

\begin{tabular}{cccc}
\hline Grupo & Comprometimento Enzimático & Mutação & Atividade in vitro \\
\hline A1 & Completo & Deleção CYP21A2 & 0 \\
& Completo & Grande conversão & 0 \\
& Completo & Del 8 nt E3 & 0 \\
& Completo & Ins T E7 & 0 \\
C2 & Q $318 X$ X & 0 \\
& Completo & Cluster E6 & 0 \\
B & Quase completo & R356W & detectável \\
C & Quase completo & I2 splice & $<2 \%$ \\
& Severo & I172N & $3-7 \%$ \\
& Moderado & V281L & $>20 \%$ \\
& Moderado & P30L & $25-50 \%$ \\
& Moderado & P453S & $66 \%$ \\
\hline
\end{tabular}

E: exon. Atividade in vitro retirada das referências Tusie-Luna e cols. (39), Higashi e cols. (40) e Helmberg e cols. (41).

Tabela 4. Genótipos mais freqüentes identificados na população brasileira com HAC-21OH e suas manifestações clínicas.

\begin{tabular}{|c|c|c|c|c|c|c|c|c|}
\hline \multirow[t]{2}{*}{ Grupos Genotípicos } & \multirow[t]{2}{*}{$\mathbf{n}$} & \multicolumn{2}{|c|}{ Sexo } & \multicolumn{3}{|c|}{ Forma } & \multirow[t]{2}{*}{ IC Diagnóstico } & \multirow{2}{*}{$\begin{array}{c}\text { Prader } \\
\text { (variação) }\end{array}$} \\
\hline & & $\mathbf{F}$ & $\mathbf{M}$ & PS & VS & NC & & \\
\hline \multicolumn{9}{|l|}{ Grupo A } \\
\hline $\mathrm{GR} / \mathrm{GR}$ & 7 & 4 & 3 & 4 & 3 & & $15 d$ & III a IV \\
\hline GR/ 12 splice & 19 & 15 & 4 & 15 & 4 & & $49 \mathrm{~d}$ & $\| \mathrm{a} \mathrm{V}$ \\
\hline 12 splice/ I2 splice & 32 & 17 & 15 & 27 & 5 & & $38 \mathrm{~d}$ & III a V \\
\hline $1172 \mathrm{~N} / 1172 \mathrm{~N}$ & 15 & 6 & 9 & 0 & 15 & & $5 a$ & $\|$ a III \\
\hline I172N/12 splice & 13 & 9 & 4 & 1 & 12 & & $5,5 a$ & III a IV \\
\hline V281L/V281L & 18 & 18 & 0 & 0 & 0 & 18 & $17 a$ & - \\
\hline $\mathrm{V} 281 \mathrm{~L}^{\circ} / \mathrm{GR}$ & 8 & 7 & 1 & 2 & 1 & 5 & $4,6 \mathrm{a}$ & III* \\
\hline
\end{tabular}

GR: grande rearranjo, d: dias, a: anos, *: identificada virilização da genitália externa apenas na forma clássica, ०: o DNA destes indivíduos não foi seqüenciado e não pode ser afastada a presença de uma mutação severa no alelo contendo a mutação V281L

atividade do genótipo. A média dos níveis da $17 \mathrm{OH}-$ progesterona no grupo A foi de $230 \pm 119 \mathrm{ng} / \mathrm{mL}$, no grupo B foi de $176 \pm 95 \mathrm{ng} / \mathrm{mL}$ e no grupo $\mathrm{C}$ foi de $16 \pm 16 \mathrm{ng} / \mathrm{mL}(\mathrm{p}<0,05)$. A média dos níveis de testosterona no grupo A foi de $242 \pm 157 \mathrm{ng} / \mathrm{mL}$, no grupo B foi de $142 \pm 75 \mathrm{ng} / \mathrm{mL}$ e no grupo $\mathrm{C}$ foi de $61 \pm 38 \mathrm{ng} / \mathrm{mL}(\mathrm{p}<0,05)$. A média do grau de virilização da genitália externa no grupo A foi Prader III, variando de Prader II a V e no grupo B foi Prader III, variando de I a V. Pacientes com genótipo do grupo A apresentaram maior grau de virilização da genitália externa, porém houve uma grande sobreposição com o grau de virilização das pacientes com genótipo do grupo B. Isto se deve provavelmente a variações individuais na sensibilidade dos tecidos aos andrógenos.

Houve uma boa correlação do genótipo com o fenótipo. Todos os pacientes com genótipo do grupo A apresentaram a forma clássica, este genótipo esteve correlacionado com a forma perdedora de sal em $77 \%$ dos casos. O genótipo do grupo B esteve correlacionado com a forma virilizante simples em $98 \%$ dos casos e o do grupo C com a forma não clássica em $92 \%$ dos casos.

Apesar da boa correlação do genótipo com o fenótipo, algumas divergências foram observadas, principalmente com a mutação I2 splice. Indivíduos homozigotos ou hemizigotos (I2 splice/deleção CYP21A2 ou grande rearranjo) apresentam principalmente a forma perdedora de sal, mas também são descritos indivíduos portadores da forma virilizante simples (10). Verificamos, nesta série, que não houve diferença na manifestação clínica entre pacientes homozigotos ou hemizigotos para esta mutação, 15/18 dos pacientes homozigotos e 15/19 dos hemizigotos apresentaram a forma perdedora de sal, e não observamos diferenças nos valores de sódio ou na época de manifestação da desidratação entre os dois grupos. Os demais casos apresentaram a forma virilizante simples e isto pode ser justificado pelo fato do maquinário celular reconhecer o sítio normal de spli cing em uma pequena proporção, mas produzindo 
quantidade de aldosterona suficiente para evitar a crise de perda de sal. Witchel e cols. (29) avaliaram 38 indivíduos portadores da mutação I2 splice em homozigose ou hemizigose e também verificaram que as manifestações clínicas variaram da forma perdedora de sal à assintomática. Na nossa amostra, nenhum indivíduo portador de um destes genótipos foi assintomático. Acreditamos que o genótipo destes últimos casos relatados na literatura deve ser conseqüente de um artefato na realização da PCR, onde neste locus é freqüente a falha da amplificação do alelo normal, fenômeno conhecido como allelic drop out (30).

Outra divergência observada foi nos pacientes portadores dos grandes rearranjos em homozigose, 3/7 indivíduos apresentaram a forma virilizante simples. Classicamente, o alelo resultante de deleção do CYP21A2 ou de conversão gênica é um gene híbrido que apresenta na sua porção 5' seqüências do pseudogene e na porção 3' seqüências do gene ativo. Em 92\% dos genes híbridos, o ponto de fusão do gene híbrido ocorre após o éxon 3 (31), tendo, portanto, este gene, a mutação del $8 \mathrm{nt}$ do exon 3 que leva à perda total da atividade da enzima. Um dos 7 pacientes teve seu gene híbrido estudado e confirmou a presença da mutação Del 8 nt. Speiser e cols. (32) identificaram uma paciente com a forma perdedora de sal, homozigota para a deleção do CYP21A2; o interessante é que esta paciente na idade adulta, após interromper as medicações, não desidratou. A explicação postulada para estes pacientes é na provável existência de uma 21 hidroxilação extra-adrenal (33).

\section{Forma não Clássica da HAC-21OH}

Os indivíduos com a forma não clássica apresentam mutações do grupo C (V281L, P453S ou P30L) em homozigose ou em heterozigose, composta com as mutações dos grupos A ou B. Como o fenótipo na HAC-21OH é conferido pelo alelo com maior atividade enzimática, verificamos se estes dois genótipos apresentaram diferenças fenotípicas. Não houve diferença na idade de início de apresentação dos sintomas entre os dois grupos, a pubarca precoce foi identificada em igual freqüência em ambos grupos. A severidade das manifestações hiperandrogênicas deve depender de outras variáveis, tais como a atividade do receptor de andrógenos e a atividade da $5 \alpha$-reductase na pele (34). Entretanto, houve uma diferença significativa entre os valores da 17OH-progesterona basal e após ACTH. A média da 17-OH progesterona basal foi de $9 \pm 6 \mathrm{ng} / \mathrm{mL}$ e de 22 $\pm 20 \mathrm{ng} / \mathrm{mL}$ nos grupos $\mathrm{C} / \mathrm{C}$ e A/C, respectivamente. A média da 17-OH progesterona após ACTH foi de 45 $\pm 32 \mathrm{ng} / \mathrm{mL}$ e de $82 \pm 27 \mathrm{ng} / \mathrm{mL}$ nos grupos $\mathrm{C} / \mathrm{C}$ e
$\mathrm{A} / \mathrm{C}$, respectivamente. Estes dados mostram uma influência do alelo A na severidade do fenótipo. Mas como houve uma grande sobreposição dos valores da 17OH-progesterona pós-ACTH entre os dois grupos genotípicos, não foi possível a discriminação entre homozigotos e heterozigotos compostos utilizando-se apenas os valores hormonais. A importância da discriminação de indivíduos com a forma não clássica e heterozigotos compostos para mutações severas é que estes podem gerar filhos com a forma clássica da deficiência da 21-hidroxilase, se o outro cônjuge for heterozigoto para uma mutação severa, cuja freqüência na população é da ordem de 1:60 (12).

Um outro fato que merece discussão é o critério diagnóstico hormonal da forma não clássica, definido por valores da $17 \mathrm{OH}$-progesterona após ACTH > 10ng/mL (4), antes dos estudos do gene CYP21A2. Analisamos, nesta amostra de pacientes com a forma não clássica e com genótipo definido, os valores basais da $17 \mathrm{OH}$-progesterona e verificamos que os valores estiveram $>5 \mathrm{ng} / \mathrm{mL}$ em $80 \%$ dos pacientes, entre 2 e $5 \mathrm{ng} / \mathrm{mL}$ em $9 \%$ e $>2 \mathrm{ng} / \mathrm{mL}$ em $11 \%$ dos pacientes. $\mathrm{O}$ menor valor da $17 \mathrm{OH}$-progesterona após ACTH foi de $17 \mathrm{ng} / \mathrm{mL}$. Azziz e cols. (35), ao analisarem pacientes com forma não clássica, observaram que a maioria apresentava valores da $17 \mathrm{OH}$-progesterona após ACTH $>15 \mathrm{ng} / \mathrm{mL}$. Em nosso estudo anterior (26) e em estudo realizado por Deneaux e cols. (36), observou-se que o menor valor da $17 \mathrm{OH}$-progesterona após ACTH é de $17 \mathrm{ng} / \mathrm{mL}$ em paciente com genótipo definido. Por outro lado, em trabalho prévio, foram identificados $2 / 59$ indivíduos heterozigotos obrigatórios para a deficiência da 21-hidroxilase, com valores da $17 \mathrm{OH}$-progesterona pós-ACTH de 11 e de $15 \mathrm{ng} / \mathrm{mL}$ (37). O seqüenciamento do gene CYP21A2 destes indivíduos não identificou outras mutações, confirmando o estado de heterozigose. Portanto, estes dados indicam que os valores da $17 \mathrm{OH}-$ progesterona após ACTH entre 10 e $17 \mathrm{ng} / \mathrm{mL}$ podem ocorrer em indivíduos heterozigotos e com forma não clássica da deficiência da 21-hidroxilase, sendo o estudo molecular do gene CYP21A2 útil nesta diferenciação.

\section{CONCLUSŌES}

As mutações mais freqüentes na população brasileira com deficiência da 21-hidroxilase são também as derivadas do pseudogene CYP2IAIP, podendo ser pesquisadas por técnicas como o PCR alelo-específico. O seqüenciamento do gene CYP21A2 deve ser reali- 
zado nos casos com genótipo não definido para identificar mutações mais raras.

Os genótipos $\mathrm{A}, \mathrm{B}$ e $\mathrm{C}$ corresponderam às formas perdedora de sal, virilizante simples e não clássica, respectivamente. A mutação $\mathrm{I} 2$ splice do grupo $\mathrm{A}$, a mais freqüente mutação na HAC-210H, foi associada principalmente à forma perdedora de sal, mas também à forma virilizante simples.

A boa correlação do genótipo com o fenótipo na HAC-21OH permite sua aplicação na prática clínica para o aconselhamento genético e no diagnóstico e tratamento pré-natal das gestações de risco para a forma clássica da HAC-210H, e após screening neonatal da HAC-21OH.

\section{REFERÊNCIAS}

1. New MI, White PC, Pang S, Dupont B, Speiser PW. The adrenal hyperplasias. In: Scriver CR, Beaudet AL, Sly $S$, Valle D, eds. The metabolic basis of inherited disease. New York: McGraw-Hill Inc, 1989. p.1881-918.

2. Morel Y, Miller WL. Clinical and molecular genetics of congenital adrenal hyperplasia due to 21-hydroxylase deficiency. In: Harris $\mathrm{H}$, Hirschhorn K, eds. Adv Hum Genet 1991;20:1-68.

3. Dewailly $D$, Vantyghem-Haudiquet MC, Buvat J, Cappoen JP, Ardaens K, Racadot A, et al. Clinical and biological phenotypes in late-onset 21-hydroxylase deficiency. J Clin Endocrinol Metab 1989;63:418-23.

4. New MI, Lorenzen F, Lerner AJ, Kohn B, Oberfield SE, Pollack MS, et al. Genotyping steroid 21 -hydroxylase deficiency: hormonal reference data. J Clin Endocrinol Metab 1983;57:320-6.

5. Carroll MC, Campbell RD, Porter RR. Mapping of steroid 21 -hydroxylase genes adjacent to complement component $\mathrm{C} 4$ genes in HLA, the major histocompatibility complex in man. Proc Natl Acad Sci USA 1985;82:5215.

6. White PC, New MI, Dupont B. Structure of human steroid 21-hydroxylase genes. Proc Natl Acad Sci USA 1986;83:5111-5.

7. Higashi Y, Yoshioka H, Yamane M, Gotoh O, Fujii-Kuriyama Y. Complete nucleotide sequence of two steroid 21hydroxylase genes tandemly arranged in human chromosome: a pseudogene and a genuine gene. Proc Natl Acad Sci USA 1986;83:2841-5.

8. Higashi Y, Tanae A, Inoue H, Fujii-Kuriyama Y. Evidence for frequent gene conversions in the steroid 21-hydroxylase (P-450c21) gene: implications for steroid 21-hydroxylase deficiency. Am J Hum Genet 1988;42:17-25.

9. Lee H. CYP21 mutations and congenital adrenal hyperplasia. Clin Genet 2001;59:293-301.

10. Speiser PW, Dupont J, Zhu D, Serrat J, Buegeleisen M, Tusié-Luna MT, et al. Disease expression and molecular genotype in congenital adrenal hyperplasia due to $21-$ hydroxylase deficiency. J Clin Invest 1992:90:584-95.
11. Wedell A, Thilén A, Ritzén EM, Stengler B. Mutational spectrum of the steroid 21-hydroxylase gene in Sweden: implications for genetic diagnosis and association with disease manifestation. J Clin Endocrinol Metab 1994; 78:1 145-52.

12. White PC, Speiser PW. Congenital adrenal hyperplasia due to 21-hydroxylase deficiency. Endocr Rev 2000;21:245-91.

13. Tusié-Luna MT, Ramírez-Jiménez S, Ordóñez-Sanchez ML, Cabello-Villegas J, Altamirano-Bustamante N, CalzadaLeón $\mathrm{R}$, et al. Low frequency of deletion alleles in patients with steroid 21-hydroxylase deficiency in a Mexican population. Human Genet 1996;98:376-9.

14. Dardis A, Bergada I, Bergada C, Rivarola M, Belgorosky A. Mutations of the steroid 21-hydroxylase gene in an Argentinian population of 36 patients with classical congenital adrenal hyperplasia. J Pediat Endocrinol Metab 1997;10: 55-61.

15. Mornet E, Crété $P$, Kuttenn F, Raux-Demay M-C, Boué J, White PC, et al. Distribution of deletions and seven point mutations on CYP21B genes in three clinical forms of steroid 21-hydroxylase deficiency. Am J Hum Genet 1991;48:79-88.

16. Ezquieta B, Oliver A, Gracia R, Gancedo PG. Analysis of steroid 21-hydroxylase gene mutations in the Spanish population. Hum Genet 1995;6:198-204.

17. Carrera P, Bordone L, Azzani T, Brunelli V, Garancini MP, Chiumello $G$, et al. Point mutations in Italian patients with classic, non-classic, and cryptic forms of steroid 21hydroxylase deficiency. Hum Genet 1996;98:662-5.

18. Araújo M, Sanches MR, Suzuki LA, Guerra Jr G, Farah SB, de-Mello MP. Molecular analysis of CYP21 and C4 genes in Brazilian families with the classical form of steroid 21-hydroxylase deficiency. Braz J Med Biol Res 1996;29:1-13.

19. Paulino LC, Araujo M, Guerra Jr G, Marini SHVL, Mello MP. Mutation distribuition and CYP21/C4 locus variability in Brazilian families with the classical form of the 21hydroxylase deficiency. Acta Paedatr 1999;88:275-83.

20. Bachega TASS, Billerbeck AEC, Madureira G, Marcondes JAM, Longui CA, Leite MV, et al. 21-Hydroxylase in Brazil. Braz J Med Biol Res 2000;33:1211-6.

21. Witchel SF, Smith $R$, Crivellaro CE, Manna TD, Dichtchekenian V, Setian N, et al. CYP2 1 mutations in Brazilians patients with 21-hydroxylase deficiency. Hum Genet 2000;106:414-9.

22. Torres N, Mello MP, Germano CMR, Elias LLK, Moreira $A C$, Castro M. Phenotype and genotype correlation of the microconversion from the CYP2 IA IP to the CYP21A2 gene in congenital adrenal hyperplasia. Braz $\mathbf{J}$ Med Biol Res 2003;36:1311-8.

23. Billerbeck AEC, Bachega TASS, Frazzatto ET, Nishi MY, Goldberg AC, Marin ML, et al. A novel missense mutation, Gly424Ser, in Brazilian patients with 21-hydroxylase deficiency. J Clin Endocrinol Metab 1999;84:2870-2.

24. Lau IF, Soardi FC, Lemos-Marini SH, Guerra Jr G, Baptista MT, de Mello MP. H28+C insertion in the CYP21 gene: a novel frameshift mutation in a Brazilian patient with the classical form of 21-hydroxylase deficiency. J Clin Endocrinol Metab 2001;86:5877-80. 
25. Billerbeck AEC, Mendonç BB, Pinto EM, Madureira G, Arnhold IJP, Bachega TASS. Three novel mutations in CYP21 gene in Brazilian patients with the classical form of 21-hydroxylase deficiency due to a founder effect. $\mathbf{J}$ Clin Endocrinol Metab 2002;87:4314-7.

26. Bachega TASS, Billerbeck AEC, Marcondes JAM, Madureira G. Arnhold IJP, Mendonca BB. Influence of different genotypes on 17-hydroxyprogesterone levels in patients with nonclassical congenital adrenal hyperplasia due to 21-hydroxylase deficiency. Clin Endocrinol 2000;52:601-7.

27. Jääskeläinen J, Levo A, Voutilainen R, Partanen J. Population-wide evaluation of disease manifestation in relation to molecular genotype in steroid 21-hydroxylase (CYP21) deficiency: good correlation in a well defined population. J Clin Endocrinol Metab 1997;82:3293-7.

28. Bachega TAAS, Billerbeck AEC, Madureira G, Marcondes JAM, Longui CA, Leite MV. Molecular genotyping in Brazilian patients with the classical and nonclassical forms of 21-hydroxylase deficiency. J Clin Endocrinol Metab 1998;83:4416-9.

29. Witchel SF, Bhamidipati DK, Hoffman EP, Cohen JB. Phenotypic heterogeneity associated with the splicing mutation in congenital adrenal hyperplasia due to $21-$ hydroxylase deficiency. J Clin Endocrinol Metab 1996;81:4081-8.

30. Day DJ, Speiser PW, Schulze E, Bettendorf M, Fitness J, Barany $F$, et al. Identification of non-amplifying CYP2 1 genes when using PCR-based diagnosis of 21-hydroxylase deficiency in congenital adrenal hyperplasia (CAH) affected pedigree. Hum Mol Genet 1996;5:203948.

31. Bachega TASS, Billerbeck AEC, Madureira G, Arnhold IJP, Medeiros MA, Marcondes JAM, et al. Low frequency of CYP21B deletions in Brazilian patients with congenital adrenal hyperplasia due to 21 -hydroxylase deficiency. Hum Hered 1999;49:9-14.

32. Speiser PW, Agdere L, Ueshiba H, White PC, New MI. Aldosterone synthesis in salt wasting congenital adrenal hyperplasia with complete absence of adrenal 21hydroxylase. N Engl J Med 1991;324:145-9.

33. Winkel CA, Casey ML, Worley RJ, Madden JD, MacDonald PC. Extaadrenal steroid 21-hydroxylase activity in woman with congenital adrenal hyperplasia due to steroid 21-hydroxylase deficiency. J Clin Endocrinol Metab 1983;56:104-7.

34. Vottero A, Stratakis CA, Ghizzoni L, Longui CA, Karl M, Chrousos GP. Androgen receptor-mediated hypersensitivity to androgens in women with nonhyperandrogenic hirsutism: skewing of X-chromosome inactivation. J Clin Endocrinol Metab 1999;84:1091-5.
35. Azziz R, Dewailly D, Owerbach D. Clinical review 56 : nonclassical adrenal hyperplasia current concepts. J Clin Endocrinol Metab 1994;78:810-5.

36. Deneux C, Tardy V, Dib A, Mornet E, Billaud L, Charran $D$, et al. Phenotype-genotype correlation in 56 women with nonclassical congenital adrenal hyperplasia due to 21-hydroxylase deficiency. J Clin Endocrinol Metab $2001 ; 86(1): 207-13$.

37. Bachega TASS, Brenlha EML, Billerbeck AEC, Marcondes JAM, Madureira G. Arnhold IJP, et al. Variable ACTHstimulated 17-hydroxyprogesterone values in 21hydroxylase deficiency carriers are not related to the different CYP21 gene mutations. J Clin Endocrinol Metab 2002;87:786-90.

38. Ordonez-Sanchez ML, Ramirez-Jimenez S, Lopez-Gutierrez AU, Riba L, Gamboa-Cardiel S, Cerrillo-Hinojosa M, et al. Molecular genetic analysis of patients carrying steroid 21-hydroxylase deficiency in the Mexican population: identification of possible new mutations and high prevalence of apparent germ-line mutations. Hum Genet 1998;102:170-7.

39. Tusie-Luna MT, Speiser PW, Dumic M, New MI, White PC. A mutation (P-30L to Leu) in CYP21 represents a potential nonclassic steroid 21-hydroxylase deficiency allele. Mol Endocrinol 1991:5:685-92.

40. Higashi Y, Hiromasa T, Tanae A, Miki T, Nakura J, Kondo $\mathrm{T}$, et al. Effects of individual mutations in the P-450(C21) pseudogene on the $\mathrm{P}-450(\mathrm{C} 21)$ activity and their distribuition in the patient genomes of congenital steroid 21-hydroxylase deficiency. J Biochem 1991;109:638-44.

41. Helmberg A, Tusie-Luna MT, Tabarelli M, Kofler R, White PC. R339H e P453S: CYP21 mutations associated with nonclassic steroid 21-hydroxylase deficiency that are not apparent gene conversions. Mol Endocrinol 1992;6:1318-22.

\section{Endereço para correspondência:}

Tânia A.S.S. Bachega

Laboratório de Hormônios e Genética Molecular LIM/42

Av. Dr. Enéas de Carvalho Aguiar 155,

Prédio dos Ambulatórios, $2^{\circ}$ andar, Bloco 6

05403-900 São Paulo, SP

e-mail: tbachega@usp.br 